\title{
Quantification of Plasmodium ex vivo drug susceptibility by flow cytometry
}

Grennady Wirjanata ${ }^{1,2}$, Irene Handayuni ${ }^{1}$, Pak Prayoga ${ }^{2}$, Dwi Apriyanti ${ }^{3}$, Ferryanto Chalfein², Boni F. Sebayang ${ }^{3}$, Steven Kho ${ }^{1}$, Rintis Noviyanti ${ }^{3}$, Enny Kenangalem ${ }^{2,4}$, Brice Campo ${ }^{5}$, Jeanne Rini Poespoprodjo ${ }^{2,4,6}$, Ric N. Price ${ }^{1,7}$ and Jutta Marfurt ${ }^{1 *}$

\begin{abstract}
Background: The emergence and spread of multidrug-resistant Plasmodium falciparum and Plasmodium vivax highlights the need for objective measures of ex vivo drug susceptibility. Flow cytometry (FC) has potential to provide a robust and rapid quantification of ex vivo parasite growth.

Methods: Field isolates from Papua, Indonesia, underwent ex vivo drug susceptibility testing against chloroquine, amodiaquine, piperaquine, mefloquine, and artesunate. A single nucleic acid stain (i.e., hydroethidine (HE) for P. falciparum and SYBR Green I (SG) for P. vivax) was used to quantify infected red blood cells by FC-based signal detection. Data derived by FC were compared to standard quantification by light microscopy (LM). A subset of isolates was used to compare single and double staining techniques.

Results: In total, 57 P. falciparum and 23 P. vivax field isolates were collected for ex vivo drug susceptibility testing. Reliable paired data between LM and FC was obtained for 88 \% (295/334) of these assays. The median difference of derived $\mathrm{IC}_{50}$ values varied from -5.4 to $6.1 \mathrm{nM}$, associated with $0.83-1.23$ fold change in $\mathrm{IC}_{50}$ values between $\mathrm{LM}$ and FC. In 15 assays (5.1\%), the derived difference of $I C_{50}$ estimates was beyond the $95 \%$ limits of agreement; in eleven assays (3.7\%), this was attributable to low parasite growth (final schizont count $<40 \%$ ), and in four assays (1.4\%) due to low initial parasitaemia at the start of assay $\left(<2000 \mu^{-1}\right)$. In a subset of seven samples, LM, single and double staining $F C$ techniques generated similar $I C_{50}$ values.
\end{abstract}

Conclusions: A single staining FC-based assay using a portable cytometer provides a simple, fast and versatile platform for field surveillance of ex vivo drug susceptibility in clinical P. falciparum and P. vivax isolates.

Keywords: Malaria, Plasmodium falciparum, Plasmodium vivax, Ex vivo drug susceptibility, Drug resistance, Flow cytometry

\section{Background}

Drug resistance remains a major obstacle to malaria control and emphasizes the importance of maintaining surveillance of anti-malarial efficacy to ensure optimal patient management and timely revision of treatment guidelines. Clinical trials are logistically difficult to conduct and their interpretation is confounded by host, parasite and drug factors. In vitro drug susceptibility

\footnotetext{
*Correspondence: jutta.marfurt@menzies.edu.au

${ }^{1}$ Global and Tropical Health Division, Menzies School of Health Research, Charles Darwin University, PO Box 41096, Casuarina,

0811 Darwin, Australia

Full list of author information is available at the end of the article
}

testing provides an useful tool for monitoring drug resistant malaria of the individual components of combination therapies and investigating potential novel anti-malarial compounds prior to clinical use [1].

Several drug susceptibility assays have been developed for anti-malarial compounds with parasite growth quantified by enzyme-linked immunosorbent assay (ELISA) [2-4], fluorometry [5], or flow cytometry (FC) [6-8]. These assays have been applied to Plasmodium falciparum laboratory and field isolates, but have been limited for Plasmodium vivax drug susceptibility testing which remains still mostly reliant on microscopic quantification of parasite maturation [9-13]. The inability to sustain $P$. 
vivax in in vitro culture results in drug testing having to be conducted on fresh isolates directly from patients with malaria; this is often undertaken in laboratories with limited resources. Quantification of parasite growth by light microscopy (LM) is relatively simple, inexpensive, and suitable for use in field settings. LM can also discriminate between different parasite stages, a feature that remains critical in quantifying short-term schizont maturation assays [10]. The marked stage-specificity of drug activity, particularly apparent for piperaquine in P. falciparum and for chloroquine in $P$. vivax assays, requires diligent attention to ensure a high proportion of early ring stages at the start of the assay [14]. However, LM has several significant shortcomings. The method requires skilled microscopists applying sustained concentration on a time-consuming task. Even when assays are performed by skilled microscopists, both inter-operator as well as intra-operator variation in parasite counts is observed, highlighting the subjective nature of the method [11]. LM is also unsuitable for medium to high throughput screening for novel drug candidates. Among the available drug susceptibility methods, FC-based approaches have the advantage of being able to identify different parasite stages and to deal with the low signal-to-noise ratio inherent with the low parasitaemia of clinical field isolates. Other colourimetric or fluorometric methods that depend on red blood cell lysis are vulnerable to auto-fluorescence which exacerbates the background noise $[15,16]$.

FC-based methods using a variety of staining and detection techniques have been developed and established for drug susceptibility testing in P. falciparum laboratory strains [17-19]. Although a simple, reagent-free assay based on the quantification of haemozoin, detected by the use of depolarizing side-scatter light filters has been reported [20,21], most of the published assays are based on the detection of double-stranded DNA of Plasmodium-infected erythrocytes since, with the exception of reticulocytes which generally account for $\leq 1 \%$ of the red blood cell mass, uninfected erythrocytes do not contain a nucleus [22]. Various nucleic acid dyes have been successfully applied to quantify parasite biomass, including Hoechst [8], SYBR Green I [18], Thiazole Orange [17], SYTO-16 [23], and hydroethidine [24]. To date, few studies have described FC-based approaches for measuring drug susceptibility in $P$. falciparum $[25,26]$ and $P$. vivax $[27,28]$ field isolates. The high capital and maintenance costs of the required hardware, the sensibility of its lasers, and the need for specifically trained personnel have also limited the applicability of the FC technology to field lab-based assays. However, the development of portable and affordable FC systems provides an excellent opportunity for facilitating and improving drug susceptibility testing in Plasmodium field isolates.
The application of FC-based methods to $P$. vivax isolates was first reported by Malleret et al. [29]. Russell and colleagues further modified this double staining method, demonstrating the feasibility of FC-based quantification of chloroquine and artesunate susceptibility in $P$. falciparum and $P$. vivax field isolates [27]. More recently, a similar approach using a combination of Hoechst 33342 and hydroethidine and a portable flow cytometer equipped with a near-UV laser has been described [28]. These studies showed good correlation between the LM- and FC-based methods. The aim of the current study was to rationalize the $\mathrm{FC}$ methods further by using a single stain technique that provides a simpler, more rapid and robust assay for higher throughput drug testing in the field.

\section{Methods}

\section{Study site and subjects}

The study was conducted at a field laboratory in Timika, Papua Province, Indonesia, a region where multidrugresistant $P$. falciparum and CQ-resistant $P$. vivax are highly prevalent [10, 30, 31]. Plasmodium species isolates were collected between 2012 and 2015, from patients with malaria attending an outpatient clinic. Patients with symptomatic malaria were recruited into the study if they had a microscopically confirmed peripheral parasitaemia with monospecies of either P. falciparum or $P$. vivax between 2000 and $80,000 \mu \mathrm{L}^{-1}$. Patients were excluded from the study when they were younger than 2 years of age, had a haemoglobin level below $7 \mathrm{~g} / \mathrm{dL}$, or had anti-malarial or antibiotic treatment during the previous month. After obtaining written informed consent, blood was collected by venepuncture. Host white blood cells (WBC) were removed by cellulose column filtration as previously described [32] and packed infected red blood cells (IRBC) were used for the ex vivo drug susceptibility assay.

\section{Ex vivo drug susceptibility assay}

Plasmodium drug susceptibility was measured using a protocol modified from the World Health Organization (WHO) microtest as described previously [12, 13]. In brief, two hundred microlitres of a $2 \%$ haematocrit blood medium mixture (BMM), consisting of RPMI 1640 medium plus $10 \%$ matched human serum (P. falciparum) or McCoy's 5A medium plus $20 \%$ matched human serum (P. vivax), was added to each well of pre-dosed drug plates containing 11 serial concentrations (two-fold dilutions) of the anti-malarials (maximum concentration shown in parentheses) CQ (2992 nM), piperaquine (PIP, $1029 \mathrm{nM})$, mefloquine (MFQ, $338 \mathrm{nM}$ ), amodiaquine (AQ, $158 \mathrm{nM}$ ), and artesunate (AS, $49 \mathrm{nM}$ ). A candle jar was used to mature the parasites at $37{ }^{\circ} \mathrm{C}$ for $35-56 \mathrm{~h}$. Incubation was stopped when $>40 \%$ of ring stage parasites had reached the mature schizont stage (i.e., $\geq 5$ 
distinct nuclei per parasite) in the drug-free control well as determined by light microscopy (LM).

\section{Anti-malarial compounds}

The anti-malarial drugs CQ, PIP, MFQ, AQ, and AS were obtained from the Worldwide Anti malarial Research Network (WWARN) QA/QC Reference Material Programme [33]. The drugs were prepared as $1 \mathrm{mg} / \mathrm{mL}$ stock solutions in distilled water (CQ, PIP, MFQ, and AQ) or $70 \%$ ethanol (AS). Drug plates were pre-dosed by diluting the compounds in $50 \%$ methanol, followed by lyophilization, and stored at $4{ }^{\circ} \mathrm{C}$. All drugs tested were assayed in duplicates. Drug plates were quality controlled by measuring drug response profiles in the CQ-resistant and CQ-sensitive laboratory strains $\mathrm{K} 1$ and FC27, respectively, using the same method.

\section{Plasmodium falciparum culture strains}

Laboratory strains $\mathrm{K} 1$ and FC27 were obtained from MR4 (BEI Resources, ATCC Manassas, Virginia, USA). The parasites were kept in continuous in vitro culture as described previously [34]. To obtain highly synchronous parasite cultures, sorbitol treatment was applied once every week as described elsewhere [35].

\section{Quantification of parasites by light microscopy}

Thick blood films made from each well were stained with $5 \%$ Giemsa solution for 30 min and examined microscopically. The number of mature schizonts per 200 asexual stage parasites was determined for each drug concentration and normalized to that of the control well.

\section{Quantification of parasites by flow cytometry Staining of IRBCs for FC}

The vital dye hydroethidine (HE, Sigma-Aldrich; excitation $_{\max } / \mathrm{emission}_{\max }=535 / 610 \mathrm{~nm}$ ) was used to stain IRBCs for P. falciparum infections and the nucleic acid dye SYBR Green I (SG, Invitrogen-Molecular Probes; excitation $_{\max } /$ emission $_{\max }=497 / 520 \mathrm{~nm}$ ) for $P$. vivax infections. HE was prepared as $10 \mathrm{mg} / \mathrm{mL}$ stock solution in dimethyl sulphoxide (DMSO) and stored at $-20{ }^{\circ} \mathrm{C}$. SG stock solution $(10,000 \times$ concentrate in DMSO $)$ was stored as per the instruction manual at $-20{ }^{\circ} \mathrm{C}$. The working solution for $\mathrm{HE}$ was made by diluting the $\mathrm{HE}$ stock solution 1:250 in phosphate buffered saline (PBS, $\mathrm{pH}$ 7.4). The $1 \times$ working solution for SG was made by diluting the SG stock solution 1:10,000 in Tris-saline buffer (TSB, pH 8.5). Staining was performed by adding $50 \mu \mathrm{L} \mathrm{HE}$ or $50 \mu \mathrm{L}$ SG working solution to pelleted IRBCs (i.e., centrifugation at $1500 \mathrm{rpm}$, for $2 \mathrm{~min}$ ) from $50 \mu \mathrm{L}$ of the $2 \%$ haematocrit BMM (final HE and SG concentration: $0.63 \mathrm{mM}$ and 1:2, respectively) for $25 \mathrm{~min}$ in the dark at $37^{\circ} \mathrm{C}$. After the incubation, IRBCs were washed (i.e., centrifugation at $1500 \mathrm{rpm}$, for $2 \mathrm{~min}$ ) once with $200 \mu \mathrm{L}$ PBS, followed by final re-suspension in $200 \mu \mathrm{L}$ PBS.

To further validate the single stain method, comparison was made with the double staining method published previously [27] using a subset of isolates obtained within the whole study. For the double staining method, $20 \mu \mathrm{L}$ of BMM from each well at harvest were transferred to an empty 96-well plate and stained with a mixture of $2 \mu \mathrm{L}$ HE $10 \mathrm{mg} / \mathrm{mL}$ solution, $30 \mu \mathrm{L}$ of SG $1 \times$ working solution, and $48 \mu \mathrm{L}$ PBS (final HE and SG concentration: $0.63 \mathrm{mM}$ and 1:3.3, respectively). The mixture was incubated for $20 \mathrm{~min}$ in the dark at room temperature. The reaction was stopped by adding $200 \mu \mathrm{L}$ PBS to all wells.

\section{Gating and quantification of parasites by $F C$}

Samples were analysed using a dual-laser (blue: $488 \mathrm{~nm}$, $50 \mathrm{~mW}$ solid state; red: $640 \mathrm{~nm}, 30 \mathrm{~mW}$ diode) $3 / 1$ emission detection configuration $\mathrm{BD}$ Accuri $\mathrm{C6}^{\mathrm{TM}}$ cytometer system equipped with a high-throughput 96-well sampler (BD Accuri CSampler ${ }^{\mathrm{TM}}$ ). The blue laser was used for the detection of both dyes (SG in FL1: $530 \pm 15 \mathrm{~nm}$; HE in FL2: $585 \pm 20 \mathrm{~nm}$ ). The gating strategy is depicted in Fig. 1. First, cells were gated according to their FSC-H/ SSC-H profile to exclude debris from the red blood cell (RBC) population. Gating was then applied on the FSC-A/FSC-H profile to differentiate cell doublets from single cells. The gated single cell population was analysed further in either the SSC-H/FL2-H (P. falciparum) or the SSC-H/FL1-H (P. vivax) dot plot profiles. The mature schizont gate was set based on the drug-free control well and applied to all drug-treated wells. Gating procedure for HE and SG double staining was applied as described by Russell et al. [27]. For data acquisition, 100,000 events were analysed. In the case of low parasitaemia (i.e., less than $0.2 \%$ ), the number of events analysed was increased to 200,000. Automated analysis was performed using the BD Accuri CFlow Sampler ${ }^{\mathrm{TM}}$ software.

\section{Statistical analysis}

Dose-response data were analysed using nonlinear regression analysis (WinNonLn 4.1; Pharsight Corporation) and the $50 \%$ inhibitory concentration $\left(\mathrm{IC}_{50}\right)$ derived using an inhibitory sigmoid maximum effect $\left(E_{\max }\right)$ model. $\mathrm{IC}_{50}$ ex vivo data were only accepted if the $E_{\max }$ and $E_{0}$ of the predicted curve were within $15 \%$ of 100 and 0 , respectively. $\mathrm{IC}_{50}$ data with a high coefficient of variation $(>100 \%)$ and/or a high sum of squared residuals $(>1)$ were also rejected.

Agreement between quantification methods was assessed by Bland-Altman analysis using log-transformed $\mathrm{IC}_{50}$ data obtained with both methods [36]. Wilcoxon signed-rank test or Student's $t$-test on log-transformed 


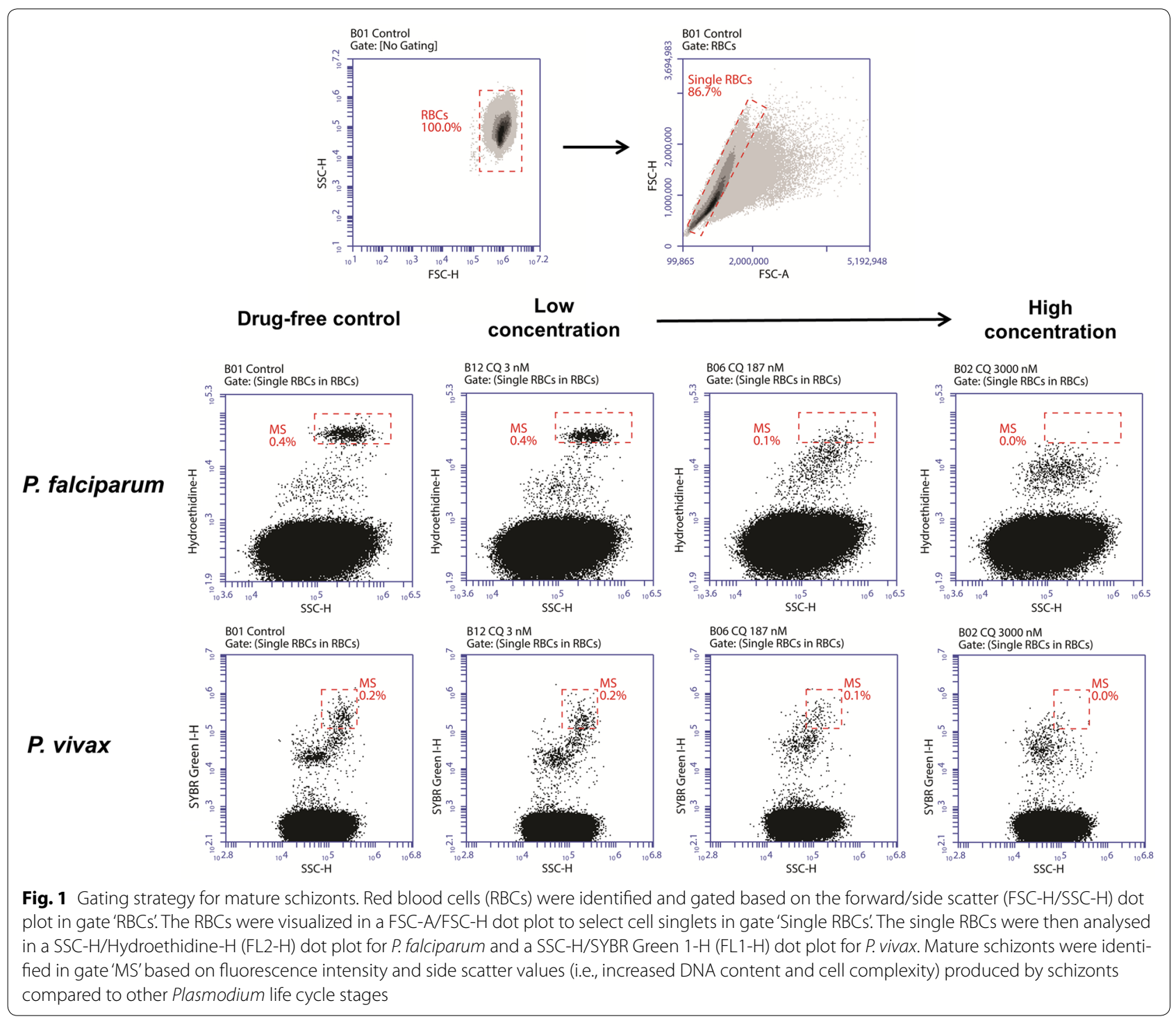

data was used to assess the difference between paired samples using the different methods. All statistical analyses were carried out using Stata (version 13.1, College Station, Texas) and GraphPad Prism software (version 6.0).

\section{Ethics}

Ethical approval for this project was obtained from the Human Research Ethics Committee of the Northern Territory Department of Health and Families and Menzies School of Health Research (HREC 2010-1396), Darwin, Australia, and the Eijkman Institute Research Ethics Commission (EIREC-47), Jakarta, Indonesia.

\section{Results}

Between May 2012 and February 2015, 57 P. falciparum and $23 P$. vivax isolates were collected and successfully harvested for the comparative analysis of flow cytometry (FC) and light microscopy (LM) based quantification. Baseline characteristics of the isolates are presented in Table 1.

Of the 334 drug assays attempted, reliable data could be derived from 329 (98.5\%) of those assessed by LM and 298 (89.2 \%) by FC. The only difference in the proportion of successful assays arose for AS, for which LM produced reliable data in $96.5 \%$ of $P$. falciparum isolates $(55 / 57)$ and $100 \%$ P. vivax isolates (23/23), compared to $66.7 \%$ $(38 / 57)$ and $73.9 \%(17 / 23)$ assays by FC, respectively (Table 2). The low success rate for the artesunate assay was associated with the first batch of plates used in which $44.1 \%(15 / 34)$ failed assays for both $P$. falciparum and $P$. vivax, whereas this failure rate fell to $15 \%(7 / 44)$ in the second batch of plates tested $(p=0.004)$. For all of the 
Table 1 Baseline characteristics of isolates for which ex vivo assays were accomplished

\begin{tabular}{|c|c|c|}
\hline Baseline characteristics & $\begin{array}{l}\text { P. falciparum } \\
\mathrm{n}=73\end{array}$ & $\begin{array}{l}P . \text { vivax } \\
\mathrm{n}=28\end{array}$ \\
\hline Total number of isolates reaching harvest (\%) & $57(78 \%)$ & $23(82 \%)$ \\
\hline Median (range) delay from venepuncture to start of culture (minutes) & $130(80-225)$ & $175(90-330)$ \\
\hline Median (range) duration of assay (hours) & $44(32-55)$ & $46(30-50)$ \\
\hline Geometric mean (95 \% Cl) parasitaemia (asexual parasites/ $\mu \mathrm{L}$ ) & $18,867(14,423-24,680)$ & $35,509(20,433-61,707)$ \\
\hline Median initial percentage (range) of parasites at ring stage & $100^{a}$ & $96(77-100)$ \\
\hline Mean $(95 \% \mathrm{Cl})$ schizont count at harvest ${ }^{\mathrm{b}}$ & $45(41-49)$ & $40(35-46)$ \\
\hline
\end{tabular}

Cl confidence interval

a No range given (all values were $100 \%$ )

b Percentage of mature schizonts per asexual blood stage parasites determined by light microscopy

Table 2 Percentage of successful assays with reliable data

\begin{tabular}{|c|c|c|c|c|c|c|}
\hline \multirow[t]{2}{*}{ Anti-malarial } & \multicolumn{3}{|c|}{ P. falciparum } & \multicolumn{3}{|c|}{$P$. vivax } \\
\hline & $n^{a}$ & Light microscopy & Flow cytometry & $\mathrm{n}^{\mathrm{a}}$ & Light microscopy & Flow cytometry \\
\hline Chloroquine & 57 & $56(98.2 \%)$ & $55(96.5 \%)$ & 23 & $23(100 \%)$ & $21(91.3 \%)$ \\
\hline Mefloquine & 39 & 39 (100 \%) & 39 (100 \%) & 17 & 17 (100 \%) & $14(82.3 \%)$ \\
\hline Piperaquine & 57 & $56(98.2 \%)$ & $55(96.5 \%)$ & 23 & $23(100 \%)$ & $21(91.3 \%)$ \\
\hline Amodiaquine & 25 & $24(96.0 \%)$ & $25(100 \%)$ & 13 & $13(100 \%)$ & $13(100 \%)$ \\
\hline Artesunate & 57 & 55 (96.5 \%) & 38 (66.7 \%) & 23 & 23 (100 \%) & 17 (73.9\%) \\
\hline
\end{tabular}

a Number of drug assays attempted

other drugs tested, reliable data could not be obtained for a total of 10 assays and this was attributable to technical errors; for nine assays, the number of acquired events by FC was too low and for one assay, microscopy slide preparation was inaccurate.

In total, 294 ( $88 \%$ ) assays could be quantified by both $\mathrm{LM}$ and $\mathrm{FC}$. The median $\mathrm{IC}_{50}$ values for all isolates with successful LM and FC assays are depicted in Fig. 2. These ex vivo drug susceptibility data are in concordance with ex vivo $\mathrm{IC}_{50}$ estimates produced in parallel studies in the area confirming a high level of CQ resistance in both Plasmodium species in Papua Indonesia [37, 38].

There were significant, albeit modest differences in $\mathrm{IC}_{50}$ values derived by $\mathrm{LM}$ and FC for PIP [median difference $=6.1 \mathrm{nM}$ (range -18.7 to $21.0 \mathrm{nM}$ ) ] and $\mathrm{AQ}$ [median difference $=2.8 \mathrm{nM}$ (range -2.7 to $15.1 \mathrm{nM}$ )], in P. falciparum isolates. No significant differences were observed between methodologies for P. vivax (Table 3).

Bland-Altman plots are presented in Fig. 3 for each drug according to the species tested and the differences in $\mathrm{IC}_{50} \mathrm{~s}$ in Table 3. From the 285 P. falciparum assays, there were 11 (3.86\%) outliers with differences in derived $\mathrm{IC}_{50} \mathrm{~s}$ up to $60 \mathrm{nM}$ for CQ, $18 \mathrm{nM}$ for PIP, $19 \mathrm{nM}$ for MFQ, $15 \mathrm{nM}$ for $\mathrm{AQ}$, and $3.6 \mathrm{nM}$ for AS. In P. vivax, there were four (3.5\%) outliers out of 115 assays with maximum values of $60 \mathrm{nM}$ for CQ, $27.5 \mathrm{nM}$ for PIP, $40 \mathrm{nM}$ for $\mathrm{AQ}$, and $5 \mathrm{nM}$ for AS. Eleven (73\%) of these outliers were identified to be attributable to isolates with a low initial parasitaemia at the start of the assays $(<2000$ parasites/ $\mu \mathrm{L})$, and four isolates due to low schizont counts $(<40 \%)$ at harvest associated with arrested trophozoite development or gametocytogenesis, ultimately resulting in suboptimal harvest.

To compare single with double staining, a subset of seven isolates (5 P. falciparum and $2 P$. vivax) were assessed by both the single staining method presented in the current study and the double staining method published by Russell et al. [27]. The results are summarized in Table 4. Overall, similar $\mathrm{IC}_{50}$ values were obtained when these methods were compared in both $P$. falciparum laboratory strains and clinical $P$. falciparum and $P$. vivax field isolates.

\section{Discussion}

Methods for quantifying ex vivo growth of Plasmodium that include parasite staging are currently limited to light microscopy (LM) and flow cytometry (FC). The present study provides a head-to-head comparison of these two approaches highlighting the utility and advantages of a single staining FC method. The assay was easy to apply in a remote field laboratory and produced results comparable to the LM-based method. Successful paired assay data 


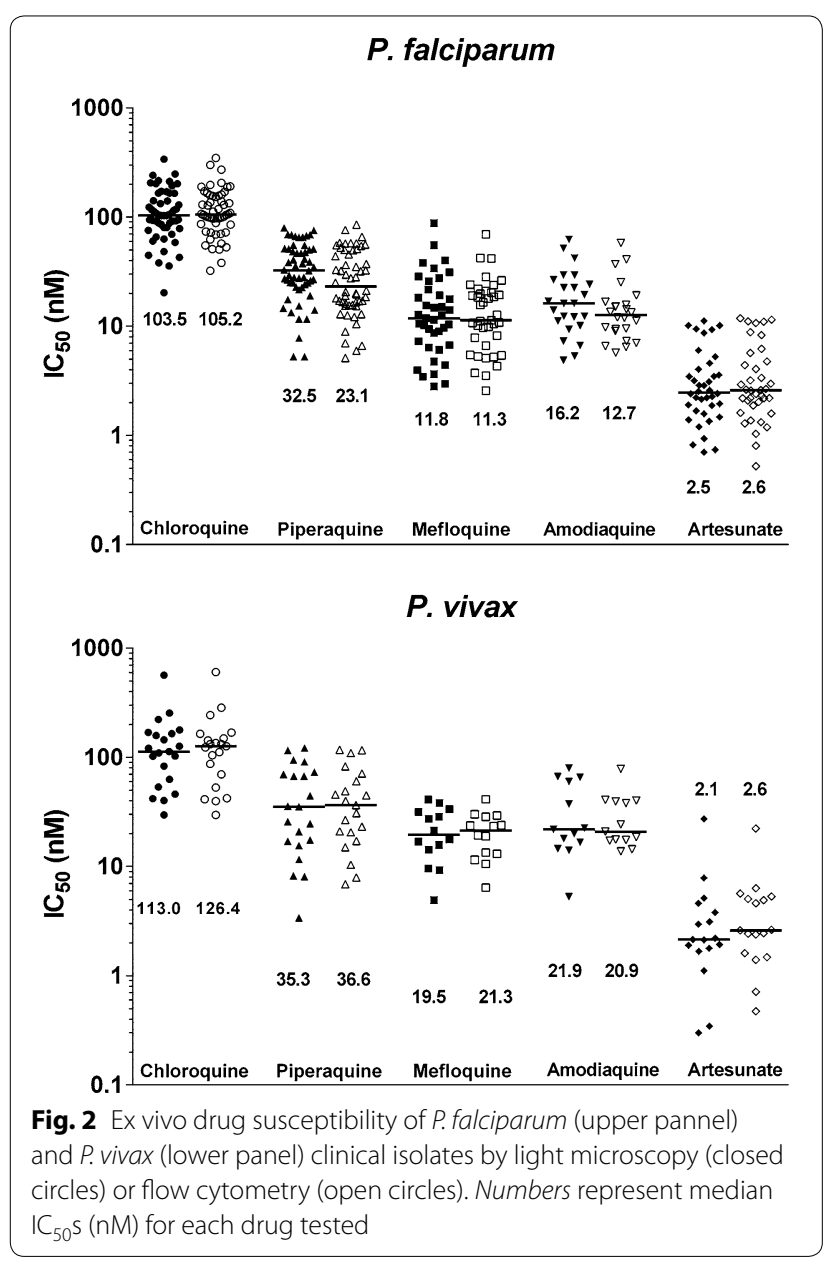

could be generated in $88 \%$ of drug assays, although the success rate was somewhat lower for AS when using FC (66.7 and $73.9 \%$ for $P$. falciparum and $P$. vivax, respectively), compared to LM (96.5 and $100 \%$ for $P$. falciparum and $P$. vivax, respectively). The failure to quantify artesunate susceptibility appeared to be related to a specific batch of drug plates that was, by mistake, stored at ambient temperature which resulted in $44.1 \%(15 / 34)$ failed assays compared to a subsequent batch in which the number of failed assay decreased to $15 \%(7 / 44)$.

During the initial phase of assay development using $P$. falciparum laboratory strains, cell staining was performed using HE. However, early experiments with P. vivax field isolates did not yield consistent results, with some isolates failing to produce sufficient fluorescent signals to distinguish both uninfected RBCs versus infected RBCs (IRBC) and the different life cycle stages; the results confounded the gating procedure for $P$. vivax isolates. Alternative nucleic acid (NA) dyes were tested (SYBR Safe nucleic acid stain, Thiazole Orange, SYTO 16, and SYBR Green I). SYBR Green I (SG) was chosen for
P. vivax since it resulted in significantly better resolution of the different RBC and IRBC populations with clear separation between schizonts and other life cycle stages. However, the HE stain produced the optimal results for $P$. falciparum isolates, with good resolution of different $\mathrm{RBC}$ and IRBC populations and higher signal-to-noise ratio compared to SG. A comparative analysis of the single staining method with the HE plus SG double staining method previously published by Russell et al. [27] was conducted in two P. falciparum laboratory strains and a subset of five $P$. falciparum and two $P$. vivax clinical field isolates summarized in Table 4. Both methods produced comparable results and appear suitable for assessing antimalarial drug susceptibility (Table 2). The advantage of using HE or SG single staining is that there is no need to run single stain controls in each experiment in order to compensate for spectral overlap that occurs when double staining methods using fluorescent dyes with close excitation/emission spectra such as HE and SG are applied. Therefore, the presented single staining method greatly facilitates raw FC data analysis.

Overall, the drug susceptibility data quantified by LM and FC were similar. Statistically significant differences in $\mathrm{IC}_{50}$ values were observed for PIP and AQ in P. falciparum. However, the median differences were modest (6.1 nM for PIP and $2.8 \mathrm{nM}$ for AQ), all within the confidence bounds of the assay and therefore, unlikely to be of biological or clinical significance. In total, there were 15 outliers with higher differences in $\mathrm{IC}_{50}$ s derived by $\mathrm{FC}$ and LM. Several factors are likely to have contributed to these outliers, the most notable of which was a reduced parasite count, either from a low starting parasitaemia (lower than 2000 parasites $/ \mu \mathrm{L}$ ), or inadequate parasite growth leading to a low final mature schizont count below $40 \%$. Quantification of low parasite densities is a recognized factor undermining the confidence of LM or FC $[10,14]$. Given the time-consuming and subjective nature of LM-based assays, those assays with reduced parasite count increase the workload on the microscopists, which leads to lower assay reading accuracy. The reliability of FC data can be improved by increasing the number of acquired events that enables greater confidence in gating the schizont population. Another potential confounding factor in the comparison of the LM and FC data is the presence of abnormal (unhealthy) schizonts, which occur following drug treatment and/or suboptimal growth. The LM-based method requires visual quantification of mature schizonts with $>5$ chromatin dots, whereas abnormal schizonts with irregular chromatin dots plus altered shape and size are not classified as mature schizonts. However, these abnormal schizonts cannot be readily distinguished with the FC method since quantification is based on side scatter properties 
P. falciparum

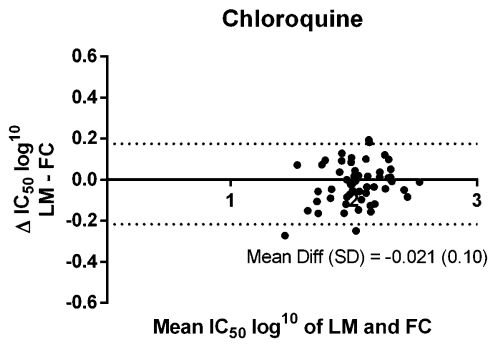

Piperaquine

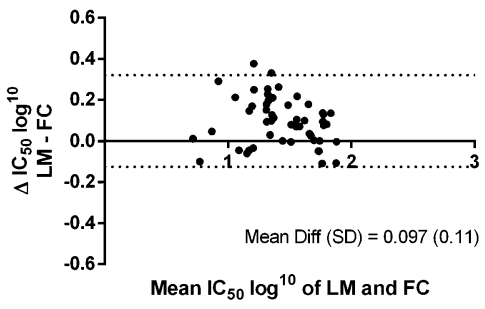

Mefloquine

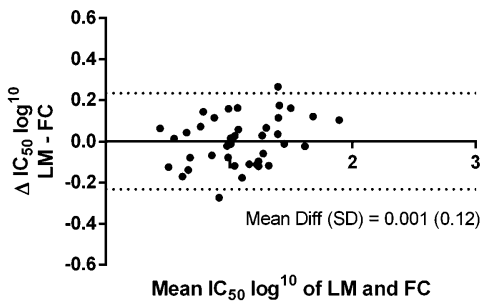

Amodiaquine

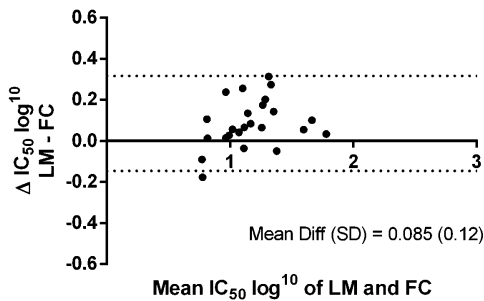

Artesunate

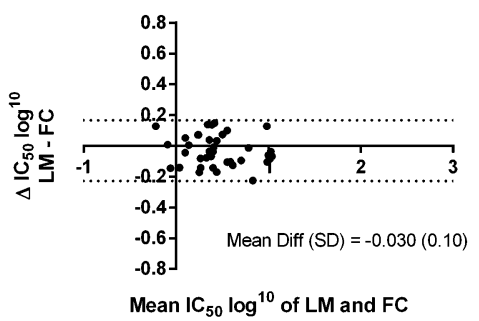

P. vivax

Chloroquine

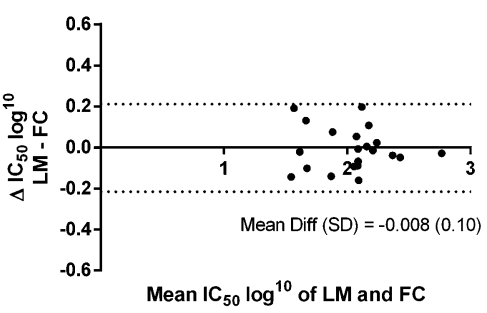

Piperaquine

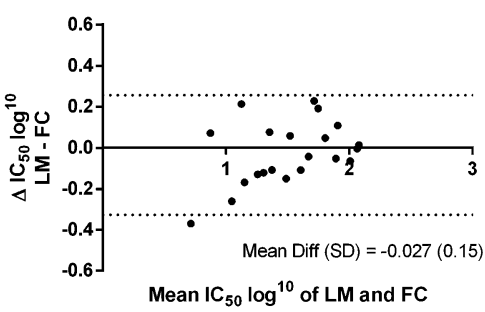

Mefloquine

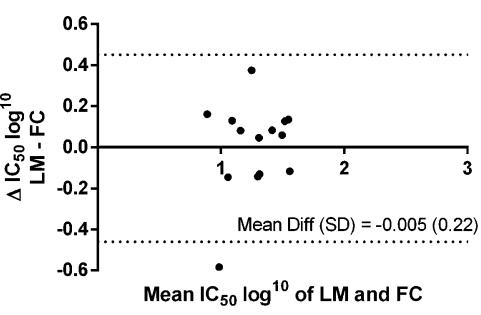

Amodiaquine

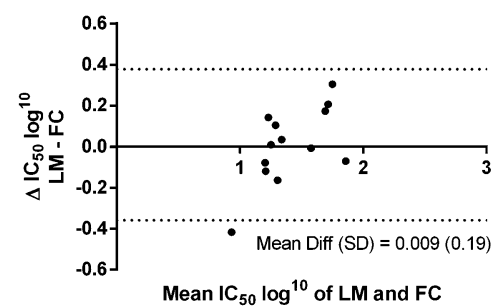

Mean IC $50 \log ^{10}$ of LM and FC

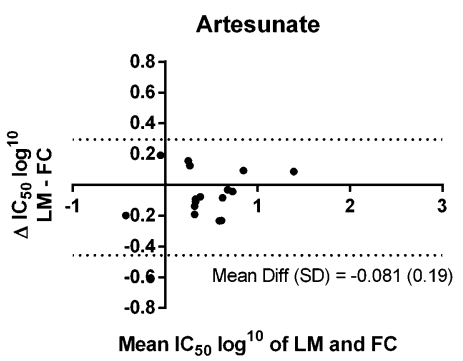

Fig. 3 Bland-Altman plots of $L M$ and $F C$ derived $I C_{50}$ s. IC $C_{50}$ values of each drug tested in $P$. falciparum (left) and P. vivax (right). Dotted lines represent the $95 \%$ limits of agreement between LM and FC 
Table 3 Difference in IC 50 estimates (nM) derived by light microscopy (LM) and flow cytometry (FC)

\begin{tabular}{|c|c|c|c|c|c|c|c|c|}
\hline \multirow[t]{2}{*}{ Anti-malarial } & \multicolumn{4}{|c|}{ P. falciparum } & \multicolumn{4}{|c|}{ P. vivax } \\
\hline & $\mathbf{n}$ & $\begin{array}{l}\text { Mean }[95 \% \mathrm{Cl}] \\
\text { difference in } \mathrm{IC}_{50} \\
\text { LM-FC (nM) }\end{array}$ & $\begin{array}{l}\text { Median [Range] } \\
\text { difference in IC } 50 \\
\text { LM-FC (nM) }\end{array}$ & $\begin{array}{l}\Delta \mathrm{IC}_{50} \mathrm{LM}-\mathrm{FC} \\
p \text { value }^{\mathrm{a}}\end{array}$ & $\mathbf{n}$ & $\begin{array}{l}\text { Mean }[95 \% \mathrm{Cl}] \\
\text { difference in IC } 50 \\
\mathrm{LM}-\mathrm{FC}(\mathrm{nM})\end{array}$ & $\begin{array}{l}\text { Median [Range] } \\
\text { difference in } I_{50} \\
\text { LM-FC (nM) }\end{array}$ & $\begin{array}{l}\Delta \mathrm{IC}_{50} \mathrm{LM}-\mathrm{FC} \\
p \text { value }^{\mathrm{a}}\end{array}$ \\
\hline Chloroquine & 54 & $-3.8[-10.7$ to 3.2$]$ & $-5.1[-60.9-59.4]$ & 0.124 & 21 & $-4.5[-16.0$ to 7.1$]$ & $-5.4[-46.1-60.2]$ & 0.725 \\
\hline Piperaquine & 54 & 6.0 [3.8 to 8.2$]$ & $6.1[-18.7-21.3]$ & $<0.001$ & 21 & $0.7[-4.6$ to 6.0$]$ & $-4.5[-15.2-27.5]$ & 0.425 \\
\hline Mefloquine & 39 & $1.3[-0.6$ to 3.1$]$ & $0.1[-5.7-18.6]$ & 0.969 & 14 & $1.2[-3.6$ to 6.0$]$ & $2.8[-13.9-15.8]$ & 0.931 \\
\hline Amodiaquine & 24 & $4.0[1.9$ to 6.0$]$ & $2.8[-2.7-15.1]$ & 0.002 & 13 & $4.7[-4.3$ to 13.7$]$ & $0.4[-11.8-40.2]$ & 0.864 \\
\hline Artesunate & 38 & $-0.4[-0.7$ to -0.01$]$ & $-0.25[-3.6-2.9]$ & 0.077 & 17 & $-0.12[-0.9$ to 0.7$]$ & $-0.45[-2.2-5.0]$ & 0.102 \\
\hline
\end{tabular}

Cl confidence interval

a $p$-values obtained from paired $t$ - test on log-transformed $\mathrm{IC}_{50}$ derived by $\mathrm{LM}$ and FC

Table 4 IC $_{50}$ values derived by different staining methods in P. falciparum laboratory strains and clinical Plasmodium isolates

\begin{tabular}{|c|c|c|c|c|c|c|c|c|c|c|c|c|}
\hline \multirow{3}{*}{$\begin{array}{l}\text { Anti-malar- } \\
\text { ial }\end{array}$} & \multicolumn{6}{|c|}{ Mean $\mathrm{IC}_{50}(\mathrm{nM})$ in P. falciparum laboratory lines ${ }^{\mathrm{a}}$} & \multicolumn{6}{|c|}{ Clinical Plasmodium isolates } \\
\hline & \multicolumn{3}{|c|}{ FC27 (CQ sensitive) } & \multicolumn{3}{|c|}{ K1 (CQ resistant) } & \multicolumn{3}{|c|}{$\begin{array}{l}\text { P. falciparum }(\mathrm{n}=5) \\
\text { Median } \mathrm{IC}_{50}(\mathrm{nM}, \text { range })\end{array}$} & \multicolumn{3}{|c|}{$\begin{array}{l}\text { P. vivax }(n=2) \\
I C_{50} \text { estimates }(n M)\end{array}$} \\
\hline & Single ${ }^{b}$ & Double $^{c}$ & $\mathrm{LM}^{\mathrm{d}}$ & Single ${ }^{\mathbf{b}}$ & Double $^{c}$ & $\mathrm{LM}^{\mathrm{d}}$ & Single ${ }^{b}$ & Double $^{c}$ & LM & Single & Double $^{c}$ & $\mathrm{LM}^{\mathrm{d}}$ \\
\hline CQ & 25.1 & 23.7 & 20.7 & 171.0 & 167.0 & 159.4 & $\begin{array}{l}85.0 \\
(52.8-165.0)\end{array}$ & $\begin{array}{l}97.3 \\
(52.9-179.0)\end{array}$ & $\begin{array}{l}108 \\
(65.6-115.0)\end{array}$ & $92.2 ; 132.0$ & $88.5 ; 150.0$ & $102.0 ; 113.0$ \\
\hline PIP & 32.8 & 32.5 & 34.7 & 63.0 & 63.9 & 89.7 & $\begin{array}{l}35.0 \\
(19.9-45.0)\end{array}$ & $\begin{array}{l}38.6 \\
(12.6-57.1)\end{array}$ & $\begin{array}{l}47.5 \\
(27.6-68.4)\end{array}$ & $79.1 ; 153.0$ & $85.6 ; 155.0$ & $91.8 ; 115.0$ \\
\hline MFQ & 46.8 & 40.9 & 49.9 & 16.6 & 14.5 & 9.4 & $\begin{array}{l}10.2 \\
(3.5-19.3)\end{array}$ & $\begin{array}{l}10.2 \\
(2.2-23.3)\end{array}$ & $\begin{array}{l}9.4 \\
(3.6-15.5)\end{array}$ & $23.0 ; 43.0$ & $30.3 ; 42.4$ & $17.7 ; 22.6$ \\
\hline AS & 4.1 & 4.3 & 2.6 & 8.7 & 7.8 & 6.0 & $2.2(1.3-3.1)$ & $2.2(1.9-3.7)$ & $1.9(1.2-2.6)$ & $2.6 ; 6.3$ & $2.8 ; 6.7$ & $3.1 ; 7.8$ \\
\hline
\end{tabular}

CQ chloroquine, PIP piperaquine, MFQ mefloquine, $A S$ artesunate

${ }^{a}$ Mean $\mathrm{IC}_{50} \mathrm{~s}$ (derived from 2 independent experiments)

b Assay quantification by flow cytometry (FC) using single staining with hydroethidine (HE) for $P$. falciparum

c Assay quantification by FC using double staining with HE and SG for both species [27, 29]

d $L M$ light microscopy

e Assay quantification by flow cytometry (FC) using single staining with SYBR Green I (SG) for P. vivax

and nucleic acid content, irrespective of the parasites' fitness.

The FC-based quantification approach offers fast, field applicable, objective and transparent post assay sample processing and data acquisition. LM-based quantification requires assay harvest, thick blood film preparation from each drug well, slide drying, Giemsa staining, followed by slide reading and cross checking of microscopy. For a standard assay of one Plasmodium isolate, testing four drugs on a 96-well format, this can be accomplished between 16 and $20 \mathrm{~h}$. The FC-based approach requires cell staining, washing, and final resuspension and data acquisition by FC, with a total average processing time of $2 \mathrm{~h}$. The assay procedure per se (i.e., sample preparation and assay duration of 52-56 h) and data analysis (i.e. pharmacodynamics modelling of raw drug response data) does not differ between the two approaches. Hence, between 14 and 18 man hours can be saved, varying according to the LM and FC skills of the operators.

The FC approach also has the advantage of requiring a smaller volume of blood than LM quantification. In a typical run, less than $0.2 \mu \mathrm{L}$ packed $\mathrm{RBCs}$ from the $2 \%$ haematocrit $\mathrm{BMM}$ is required for each assay. A reduction in the blood volume required for each drug assay should increase the number of drugs that can be tested in parallel from the same volume of blood. The savings in processing costs and human resources counter balance the higher initial costs for hardware and maintenance for FC and make this a viable field adapted technology.

\section{Conclusion}

The single staining FC-based quantification of ex vivo parasite growth provides a rapid and reliable estimate of 
drug response. Its implementation in field laboratories to replace or complement the time-consuming and labourintensive LM-based method has potential to greatly facilitate surveillance and screening of anti-malarial agents.

\begin{abstract}
Abbreviations
AQ: amodiaquine; AS: artesunate; BMM: blood medium mixture; CQ: chloroquine; ELISA: enzyme-linked immunosorbent assay; FC: flow cytometry; HE: hydroethidine; $I_{50}$ : half-maximal inhibition of growth; IRBC: infected red blood cells; WBC: white blood cell; LM: light microscopy; MMV: Medicines for Malaria Venture; MFQ: mefloquine; PIP: piperaquine; RBC: red blood cells; SG: SYBR Green I; WHO: World Health Organization; WWARN: WorldWide Antimalarial Resistance Network.
\end{abstract}

\section{Authors' contributions}

Conceived and designed the study: GW, RNP, JM. Performed field laboratory experiments and corresponding QC procedures: GW, IH, PP, DA, FC, BFS, SK. Analysed the data: GW, IH, JM. Contributed reagents, materials, and analysis tools: RN, EK, BC, JRP. Wrote the paper: GW, RNP, JM. All authors read and approved the final manuscript.

\section{Author details}

${ }^{1}$ Global and Tropical Health Division, Menzies School of Health Research, Charles Darwin University, PO Box 41096, Casuarina, 0811 Darwin, Australia. 2 Papuan Health and Community Development Foundation (PHCDF), Timika, Papua, Indonesia. ${ }^{3}$ Eijkman Institute for Molecular Biology, Jl. Diponegoro 69, Jakarta 10430, Indonesia. ${ }^{4}$ District Health Authority, Timika, Papua, Indonesia. ${ }^{5}$ Medicines for Malaria Venture (MMV), 20 rte de Pré-Bois, PO Box 1826, 1215 Geneva 15, Switzerland. ${ }^{6}$ Department of Paediatrics, Faculty of Medicine, Gadjah Mada University, Yogyakarta, Indonesia. ${ }^{7}$ Nuffield Department of Clinical Medicine, Centre for Tropical Medicine and Global Health, University of Oxford, Oxford, UK.

\section{Acknowledgements}

We are grateful to Lembaga Pengembangan Masyarakat Amungme Kamoro, the staff of the Rumah Sakit Mitra Masyarakat (RSMM) Hospital, and Dr Paulus Sugiarto for their support in conducting this study. We thank the Australian Red Cross blood transfusion service for the supply of human sera. The study was funded by the Wellcome Trust (Senior Research Fellowship in Clinical Science 091625 to RNP, Research Training Fellowship 099875 to JRP), the National Health and Medical Research Council (Project Grant 1023438 to RNP and JM), the Swiss National Science Foundation (Fellowship for Advanced Researchers to JM), and Medicines for Malaria Venture (MMV), Geneva, Switzerland.

\section{Competing interests}

Brice Campo is an employee of MMV. The other authors declare that they have no competing interests.

Received: 17 August 2015 Accepted: 11 October 2015

Published online: 24 October 2015

\section{References}

1. Desjardins RE, Canfield CJ, Haynes JD, Chulay JD. Quantitative assessment of antimalarial activity in vitro by a semiautomated microdilution technique. Antimicrob Agents Chemother. 1979;16:710-8.

2. Noedl H, Bronnert J, Yingyuen K, Attlmayr B, Kollaritsch H, Fukuda M. Simple histidine-rich protein 2 double-site sandwich enzyme-linked immunosorbent assay for use in malaria drug sensitivity testing. Antimicrob Agents Chemother. 2005;49:3575-7.

3. Noedl H, Yingyuen K, Laoboonchai A, Fukuda M, Sirichaisinthop J, Miller RS. Sensitivity and specificity of an antigen detection ELISA for malaria diagnosis. Am J Trop Med Hyg. 2006;75:1205-8.

4. Kaddouri H, Nakache S, Houzé S, Mentré F, Le Bras J. Assessment of the drug susceptibility of Plasmodium falciparum clinical isolates from africa by using a Plasmodium lactate dehydrogenase immunodetection assay and an inhibitory maximum effect model for precise measurement of the 50-percent inhibitory concentration. Antimicrob Agents Chemother 2006;50:3343-9.

5. Smilkstein M, Sriwilaijaroen N, Kelly JX, Wilairat P, Riscoe M. Simple and inexpensive fluorescence-based technique for high-throughput antimalarial drug screening. Antimicrob Agents Chemother. 2004;48:1803-6.

6. Van Vianen PH, Thaithong S, Reinders PP, Van Engen A, Van Der Keur M, Tanke HJ, et al. Automated flow cytometric analysis of drug susceptibility of malaria parasites. Am J Trop Med Hyg. 1990;43:602-7.

7. Pattanapanyasat K, Thaithong S, Kyle DE, Udomsangpetch R, Yongvanitchit K, Hider RC, Webster HK. Flow cytometric assessment of hydroxypyridinone iron chelators on in vitro growth of drug-resistant malaria. Cytometry. 1997;27:84-91.

8. Grimberg BT, Erickson JJ, Sramkoski RM, Jacobberger JW, Zimmerman PA. Monitoring Plasmodium falciparum growth and development by UV flow cytometry using an optimized Hoechst thiazole orange staining strategy. Cytometry A. 2008;73:546-54.

9. Russell BM, Udomsangpetch R, Rieckmann KH, Kotecka BM, Coleman RE, Sattabongkot J. Simple in vitro assay for determining the sensitivity of Plasmodium vivax isolates from fresh human blood to antimalarials in areas where P. vivax is endemic. Antimicrob Agents Chemother. 2003;47:170-3.

10. Russell B, Chalfein F, Prasetyorini B, Kenangalem E, Piera K, Suwanarusk R, et al. Determinants of in vitro drug susceptibility testing of Plasmodium vivax. Antimicrob Agents Chemother. 2008;52:1040-5.

11. Russell B, Suwanarusk R, Malleret B, Costa FT, Snounou G, Baird JK, et al. Human ex vivo studies on asexual Plasmodium vivax: the best way forward. Int J Parasitol. 2012;42:1063-70.

12. Marfurt J, Chalfein F, Prayoga P, Wabiser F, Wirjanata G, Sebayang B, et al. Comparative ex vivo activity of novel endoperoxides in multidrug-resistant plasmodium falciparum and P. vivax. Antimicrob Agents Chemother 2012;56:5258-63.

13. Marfurt J, Chalfein F, Prayoga P, Wabiser F, Kenangalem E, Piera KA, et al. Ex vivo drug susceptibility of ferroquine against chloroquine-resistant isolates of Plasmodium falciparum and P. vivax. Antimicrob Agents Chemother. 2011;55:4461-4.

14. Kerlin DH, Boyce K, Marfurt J, Simpson JA, Kenangalem E, Cheng Q, et al. An analytical method for assessing stage-specific drug activity in Plasmodium vivax malaria: implications for ex vivo drug susceptibility testing. PLoS Neglect Trop Dis. 2012;6:e1772.

15. Druilhe P, Brasseur P, Blanc C, Makler M. Improved assessment of Plasmodium vivax response to antimalarial drugs by a colorimetric double-site plasmodium lactate dehydrogenase antigen capture enzyme-linked immunosorbent assay. Antimicrob Agents Chemother. 2007;51:2112-6.

16. Kosaisavee V, Suwanarusk R, Nosten F, Kyle DE, Barrends M, Jones J, et al. Plasmodium vivax: isotopic, PicoGreen, and microscopic assays for measuring chloroquine sensitivity in fresh and cryopreserved isolates. Exp Parasitol. 2006;114:34-9.

17. Makler MT, Lee LG, Recktenwald D. Thiazole orange: a new dye for Plasmodium species analysis. Cytometry. 1987;8:568-70.

18. Izumiyama S, Omura M, Takasaki T, Ohmae H, Asahi H. Plasmodium falciparum: development and validation of a measure of intraerythrocytic growth using SYBR Green I in a flow cytometer. Exp Parasitol. 2009;121:144-50.

19. Jouin H, Daher W, Khalife J, Ricard I, Puijalon OM, Capron M, Dive D. Double staining of Plasmodium falciparum nucleic acids with hydroethidine and thiazole orange for cell cycle stage analysis by flow cytometry. Cytometry A. 2004;57:34-8.

20. Rebelo M, Sousa C, Shapiro HM, Mota MM, Grobusch MP, Hanscheid T. A novel flow cytometric hemozoin detection assay for real-time sensitivity testing of Plasmodium falciparum. PLoS One. 2013;8:e61606.

21. Rebelo M, Tempera C, Fernandes JF, Grobusch MP, Hanscheid T. Assessing anti-malarial drug effects ex vivo using the haemozoin detection assay. Malar J. 2015;14:140.

22. Grimberg BT. Methodology and application of flow cytometry for investigation of human malaria parasites. J Immunol Meth. 2011;367:1-16.

23. Jiménez-Díaz MB, Mulet T, Gomez V, Viera S, Alvarez A, Garuti H, et al. Quantitative measurement of Plasmodium-infected erythrocytes in murine models of malaria by flow cytometry using bidimensional assessment of SYTO-16 fluorescence. Cytometry A. 2009;75:225-35. 
24. Van der Heyde HC, Elloso MM. Use of hydroethidine and flow cytometry to assess the effects of leukocytes on the malarial parasite Plasmodium falciparum. Clin Diagn Lab Immunol. 1995;2:417-25.

25. Karl S, Wong RP, St Pierre TG, Davis TM. A comparative study of a flowcytometry-based assessment of in vitro Plasmodium falciparum drug sensitivity. Malar J. 2009;8:294.

26. Woodrow CJ, Wangsing C, Sriprawat K, Christensen P, Nosten F, Rénia L, et al. A comparison between flow cytometry, microscopy and lactate dehydrogenase ELISA for Plasmodium falciparum drug-susceptibility testing under field conditions. J Clin Microbiol. 2015;53:3296-303.

27. Russell B, Malleret B, Suwanarusk R, Anthony C, Kanlaya S, Lau Y, et al. Field-based flow cytometry for ex vivo characterization of Plasmodium vivax and P. falciparum antimalarial sensitivity. Antimicrob Agents Chemother. 2013;57:5170-4.

28. Suwanarusk R, Russell B, Ong A, Sriprawat K, Chu CS, PyaePhyo A, et al. Methylene blue inhibits the asexual development of vivax malaria parasites from a region of increasing chloroquine resistance. J Antimicrobial Chemother. 2014; dku326.

29. Malleret B, Claser C, Ong ASM, Suwanarusk R, Sriprawat K, Howland SW, et al. A rapid and robust tri-color flow cytometry assay for monitoring malaria parasite development. Sci Rep. 2011;1:118.

30. Karyana M, Burdarm L, Yeung S, Kenangalem E, Wariker N, Maristela R, et al. Malaria morbidity in Papua Indonesia, an area with multidrug resistant Plasmodium vivax and Plasmodium falciparum. Malar J. 2008;7:148.

31. Ratcliff A, Siswantoro H, Kenangalem E, Wuwung M, Brockman A, Edstein $M D$, et al. Therapeutic response of multidrug-resistant Plasmodium falciparum and P. vivax to chloroquine and sulfadoxine-pyrimethamine in southern Papua, Indonesia. Trans R Soc Trop Med Hyg. 2007;101:351-9.
32. Venkatesan M, Amaratunga C, Campino S, Auburn S, Koch O, Lim P, et al Using CF11 cellulose columns to inexpensively and effectively remove human DNA from Plasmodium falciparum-infected whole blood samples. Malar J. 2012;11:41.

33. Lourens C, Watkins WM, Barnes KI, Sibley CH, Guerin PJ, White NJ, et al. Implementation of a reference standard and proficiency testing programme by the World Wide Antimalarial Resistance Network (WWARN). Malar J. 2010;9:375.

34. Trager W, Jensen JB. Human malaria parasites in continuous culture. Science. 1976;193:673.

35. Lambros C, Vanderberg JP. Synchronization of Plasmodium falciparum erythrocytic stages in culture. J Parasitol. 1979;65:418-20.

36. Bland JM, Altman DG. Statistical methods for assessing agreement between two methods of clinical measurement. Lancet. 1986;1:307-10.

37. Wirjanata G, Sebayang BF, Chalfein F, Prayoga, Handayuni I, Noviyanti R, et al. Contrasting ex vivo efficacies of "reversed chloroquine" compounds in chloroquine-resistant Plasmodium falciparum and P. vivax isolates. Antimicrob Agents Chemother. 2015;59:5721-6.

38. Wirjanata G, Sebayang BF, Chalfein F, Prayoga, Handayuni I, Trianty L, et al. Potent ex vivo activity of naphthoquine and methylene blue against drug-resistant clinical isolates of Plasmodium falciparum and Plasmodium vivax. Antimicrob Agents Chemother. 2015;59:6117-24.

\section{Submit your next manuscript to BioMed Central and take full advantage of:}

- Convenient online submission

- Thorough peer review

- No space constraints or color figure charges

- Immediate publication on acceptance

- Inclusion in PubMed, CAS, Scopus and Google Scholar

- Research which is freely available for redistribution

Submit your manuscript at

www.biomedcentral.com/submit

C Biomed Central 